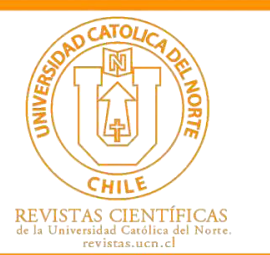

\title{
Bounds for neighborhood Zagreb index and its explicit expressions under some graph operations
}

\author{
Sourav Mondal ${ }^{1}$ (1) orcid.org/0000-0003-1928-7075 \\ Muhammad Arfan Ali² (o orcid.org/0000-0003-3148-6659 \\ Nilanjan De ${ }^{3}$ (D) orcid.org/0000-0001-9143-7045 \\ Anita $\mathrm{Pal}^{4}$
}

${ }^{1}$ National Institute of Technology Durgapur, Dept. of mathematics, Durgapur, WB, India.
souravmath94@gmail.com
${ }^{2}$ Virtual University of Pakistan, Dept. of Mathematics, Pakistan
arfanali709@gmail.com
${ }^{3}$ Calcutta Institute of Engineeringand Management, Dept. of Basic Sci. \& Humanities, Kolkata, WB, India.
de.nilanjan@rediffmail.com
${ }^{4}$ National Institute of Technology Durgapur, Dept. of mathematics, Durgapur, WB, India
anita.buie@gmail.com

Received: February 2020 | Accepted: May 2020

\begin{abstract}
:
Topological indices are useful in QSAR/QSPR studies for modeling biological and physiochemical properties of molecules. The neighborhood Zagreb index (MN) is a novel topological index having good correlations with some physiochemical properties. For a simple connected graph $G$, the neighborhood Zagreb index is the totality of square of $\delta G(v)$ over the vertex set, where $\delta G(v)$ is the total count of degrees of all neighbors of $V$ in $G$. In this report, some bounds are established for the neighborhood Zagreb index. Some explicit expressions of the index for some graph operations are also computed, which are used to obtain the index for some chemically significant molecular graphs.
\end{abstract}

Keywords:Topological index; First Zagreb index; Second Zagreb index; Neighbouhood Zagreb index; Graph operations.

MSC (2020): 05C35, 05C07, 05C40.

\section{Cite this article as (IEEE citation style):}

S. Mondal, M. A. Ali, N. De and A. Pal, "Bounds for neighborhood Zagreb index and its explicit expressions under some graph operations", Proyecciones (Antofagasta, On line), vol. 39, no. 4, pp. 799-819, Aug. 2020, doi: 10.22199/issn.0717-6279-2020-040050.

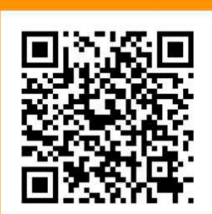

Article copyright: (C) 2020 Sourav Mondal, Muhammad Arfan Ali, Nilanjan De and Anita Pal. This is an open access article distributed under the terms of the Creative Commons License, which permits unrestricted use and distribution provided the original author and source are credited. 


\section{Introduction}

In theoretical chemistry molecular graphs $[19,12]$ are often used to model different molecular structures. Molecular graphs are actually a graphical representation of molecular structure through vertices and edges so that each vertex corresponds to atoms and the edges represent the bonds between them. Throughout this article, we consider only the molecular graph. Let $V(G)$ and $E(G)$ be the vertex and edge sets of a graph $G$ respectively. Here we use the notation $V\left(G_{i}\right), E\left(G_{i}\right), n_{i}$ and $m_{i}$ for vertex set, edge set, order and size of $G_{i}$ respectively. Here $\delta_{G}(v)$ represents the totality of degrees of all neighbors of $v$ in $G$. By neighbors of $v$, we mean the vertices that are connected to $v . K_{n}$ and $C_{n}$ represent complete and cycle graph with $n$ vertices respectively. By $\bar{G}$, we mean the complement of any graph G.

Graph theory provides an important tool called topological index to correlate the physiochemical behavior of chemicals with their molecular structure. Topological index is nothing but a numeric amount gotten from molecular graphs that describes the topology of the molecular graph and is invariant for isomorphic graphs. It is widely used in various fields of chemistry, biochemistry and nanotechnology in isomer discrimination, QSAR, QSPR and pharmaceutical medication plan and so forth. Utilization of such indices in chemistry and biology started in 1947 when chemist Harold Wiener [20] presented the wiener index for searching boiling points of alkane. One of the most well used topological indices is the Zagreb index first introduced by Gutman and Trinajestić [13], where they investigated the total $\pi$-electron energy dependency on molecular structure.

The first Zagreb index $M_{1}(G)$ and the second Zagreb index $M_{2}(G)$ for a molecular graph $(G)$ are as follows:

$$
\begin{gathered}
M_{1}(G)=\sum_{v \in V(G)} \operatorname{deg}_{G}(v)^{2}=\sum_{u v \in E(G)}\left[\operatorname{deg}_{G}(u)+\operatorname{deg}_{G}(v)\right] \\
M_{2}(G)=\sum_{u v \in E(G)} \operatorname{deg}_{G}(u) \operatorname{deg}_{G}(v)
\end{gathered}
$$

Following first Zagreb index, we introduced a novel topological index named as neighborhood Zagreb index $\left(M_{N}\right)$ [16]. To determine the usefulness of a topological index to predict physiochemical behavior of chemical 
compound, we compute correlation coefficient $(r)$ between physiochemical properties and topological indices. Topological indices for which $r^{2}$ is greater than or equal to 0.8 is very useful in QSPR/QSAR analysis. In [16], it is shown that the correlations of neighborhood Zagreb index with Entropy $\left(r^{2}=0.98915\right)$ and Acentric factor $\left(r^{2}=0.907460\right)$ are excellent. Thus $M_{N}$ can predict Entropy and Acentric factor with power full accuracy. The above correlations ensure the compatibility of the index in QSPR/QSAR analysis. A disadvantage of most topological indices is degeneracy, i.e. more than one isomer have same index. In case octane isomers, $M_{N}$ index yields well response (mean isomer degeneracy $=1$ ) [16]. It confirms the isomer-discrimination ability of this index with high accuracy. Consequently it is worth to discuss the mathematical properties of this chemically interesting index.

In $[2,4,7]$, different bounds for topological descriptors are discussed. Analytical expressions of topological indices under some graph operations are derived in $[1,6,8,15]$. In this regard we obtain some bounds of the neighborhood Zagreb index and compute some exact expressions for the index under some graph operations. Also we apply them on some composite and chemically interesting graphs.

\section{Some bounds of neighborhood Zagreb index}

Throughout this section we obtain some upper and lower bounds of the neighborhood Zagreb index using some standard inequalities.

Lemma 1. For a graph $G$, we have

(i) $\sum_{i=1}^{n} \delta_{G}\left(v_{i}\right)=M_{1}(G)$,

(ii) $\sum_{i=1}^{n} \delta_{G}\left(v_{i}\right) \operatorname{deg}_{G}\left(v_{i}\right)=2 M_{2}(G)$.

Lemma 2. (Quadratic mean $\geq$ Arithmetic mean) For $n$ positive numbers $x_{1}, x_{2}, \ldots, x_{n}$, we have

$$
\sqrt{\frac{x_{1}^{2}+x_{2}^{2}+\ldots+x_{n}^{2}}{n}} \geq \frac{x_{1}+x_{2}+\ldots+x_{n}}{n},
$$

where equality holds iff $x_{1}=x_{2}=\ldots=x_{n}$. 
Proposition 1. For a graph $G$ with $n$ vertices, we have

$$
M_{N}(G) \geq \frac{M_{1}(G)^{2}}{n}
$$

where equality holds iff $G$ is regular.

Proof 1. Considering $x_{i}=\delta_{G}\left(v_{i}\right)$ for $i=1,2, \ldots, n$, inequality (2.1) becomes

$$
\sqrt{\frac{\delta_{G}\left(v_{1}\right)^{2}+\delta_{G}\left(v_{2}\right)^{2}+\ldots+\delta_{G}\left(v_{n}\right)^{2}}{n}} \geq \frac{\delta_{G}\left(v_{1}\right)+\delta_{G}\left(v_{2}\right)+\ldots+\delta_{G}\left(v_{n}\right)}{n}
$$

Using lemma 1 and the definition of $M_{N}$ index, we have from (2.2),

$$
\sqrt{\frac{M_{N}(G)}{n}} \geq \frac{M_{1}(G)}{n},
$$

after squaring both sides of (2.4) we get the required result.

The equality in (2.2) holds iff $\delta_{G}\left(v_{1}\right)=\delta_{G}\left(v_{2}\right)=\ldots=\delta_{G}\left(v_{n}\right)$. Hence equality in (2.2) holds iff $G$ is regular.

Lemma 3. (Cauchy-Schwartz inequality) [14] Let $x_{i}$ and $y_{i}$ be real numbers for all $1 \leq i \leq n$. Then

$$
\left(\sum_{i=1}^{n} x_{i} y_{i}\right)^{2} \leq\left(\sum_{i=1}^{n} x_{i}^{2}\right)\left(\sum_{i=1}^{n} y_{i}^{2}\right)
$$

Equality holds iff $x_{i}=k y_{i}$ for some constant $k$ and for each $1 \leq i \leq n$.

Proposition 2. For a graph $G$, we have

$$
M_{N}(G) \geq \frac{4 M_{2}(G)^{2}}{M_{1}(G)}
$$

where equality holds iff $\delta_{G}\left(v_{i}\right)=k \operatorname{deg}_{G}\left(v_{i}\right)$ for some constant $k$ and for each $1 \leq i \leq n$.

Proof 2. . For each $i=1,2, \ldots, n$, putting $x_{i}=\delta_{G}\left(v_{i}\right)$ and $y_{i}=\operatorname{deg}_{G}\left(v_{i}\right)$ in (2.5), we have

$$
\left(\sum_{i=1}^{n} \delta_{G}\left(v_{i}\right) \operatorname{deg}_{G}\left(v_{i}\right)\right)^{2} \leq \sum_{i=1}^{n} \delta_{G}\left(v_{i}\right)^{2} \sum_{i=1}^{n} \operatorname{deg}_{G}\left(v_{i}\right)^{2},
$$


applying lemma 1 and the definition of $M_{N}$ and $M_{1}$ index, we can rewrite the inequality (2.7) as

$$
4 M_{2}(G)^{2} \leq M_{N}(G) M_{1}(G),
$$

which gives the inequality (2.6).

Equality in (2.7) holds iff $\delta_{G}\left(v_{i}\right)=k \operatorname{deg}_{G}\left(v_{i}\right)$ for some constant $k$ and for each $1 \leq i \leq n$. Hence Equality in (2.6) holds iff $\delta_{G}\left(v_{i}\right)=k \operatorname{deg}_{G}\left(v_{i}\right)$ for some constant $k$ and for each $1 \leq i \leq n$.

Lemma 4. (Bhatia and Davis's bound on variance) [3] Let $x_{1}, x_{2}, \ldots, x_{n}$ be real numbers such that $m \leq x_{i} \leq M$ for all $1 \leq i \leq n$ and $\mu=\frac{\sum_{i=1}^{n} x_{i}}{n}$. Then

$$
\frac{\sum_{i=1}^{n}\left(x_{i}-\mu\right)^{2}}{n} \leq(M-\mu)(\mu-m),
$$

where equality holds iff each $x_{i}$ is either $M$ or $m$.

If we consider $x_{i}=\delta_{G}\left(v_{i}\right)$ for each $i(=1,2, \ldots, n), m=\delta_{N}$ and $M=\Delta_{N}$, then $\mu=\frac{M_{1}(G)}{n}$ and inequality (2.9) gives the following bound.

Proposition 3. Let $G$ be a graph with $n$ vertices. Also consider

$$
\begin{gathered}
\Delta_{N}=\max \left\{\delta_{G}(v): v \in V(G)\right\}, \\
\delta_{N}=\min \left\{\delta_{G}(v): v \in V(G)\right\} .
\end{gathered}
$$

Then we have

$$
M_{N}(G) \leq \frac{2}{n} M_{1}(G)^{2}+\left(\delta_{N}+\Delta_{N}\right) M_{1}(G)-n \delta_{N} \Delta_{N},
$$

where equality holds iff each $\delta_{G}\left(v_{i}\right)(1 \leq i \leq n)$ is either $\delta_{N}$ or $\Delta_{N}$.

Lemma 5. (Diaz-Metcalf inequality)[9] Let $x_{i}$ and $y_{i}$ be two sequence of real numbers with $x_{i} \neq 0(i=1,2, \ldots, n)$ and such that $m x_{i} \leq y_{i} \leq M y_{i}$, then we have

$$
\sum_{i=1}^{n} y_{i}^{2}+m M \sum_{i=1}^{n} x_{i}^{2} \leq(M+m) \sum_{i=1}^{n} x_{i} y_{i}
$$

where equality holds iff either $y_{i}=m x_{i}$ or $y_{i}=M x_{i} \forall i=1,2, \ldots, n$. 
If we consider $x_{i}=\operatorname{deg}_{G}\left(v_{i}\right), y_{i}=\delta_{G}\left(v_{i}\right), m=1, M=\Delta_{N}$, inequality (2.11) yields the following bound.

Proposition 4. Let $G$ be a graph with $\Delta_{N}=\max \left\{\delta_{G}(v): v \in V(G)\right\}$, then we have

$$
M_{N}(G) \leq 2\left(\Delta_{N}+1\right) M_{2}(G)-\Delta_{N} M_{1}(G),
$$

where equality holds iff $\delta_{G}\left(v_{i}\right)=\operatorname{deg}_{G}\left(v_{i}\right)$ or $\delta_{G}\left(v_{i}\right)=\Delta_{N} \operatorname{deg}_{G}\left(v_{i}\right) \forall i=$ $1,2, \ldots, n$.

Lemma 6 (18). Let $\left(x_{1}, x_{2}, \ldots, x_{n}\right)$ be positive $n$-tuple such that there exists positive numbers $A$, a satisfying $0 \leq a \leq x_{i} \leq A$, then we have

$$
\frac{n \sum_{i=1}^{n} x_{i}^{2}}{\left(\sum_{i=1}^{n} x_{i}\right)^{2}} \leq \frac{1}{4}\left(\frac{\sqrt{A}}{\sqrt{a}}+\frac{\sqrt{a}}{\sqrt{A}}\right)^{2}
$$

where equality holds iff $a=A$ or $q=\frac{\frac{A}{a}}{\frac{A}{a}+1} n$ is an integer and $q$ of the numbers $x_{i}$ coincide with $a$ and the remaining $(n-q)$ of the $x_{i}^{\prime} s$ coincide with $A(\neq a)$.

Considering $x_{i}=\delta_{G}\left(v_{i}\right), a=\delta_{N}$ and $A=\Delta_{N}$ in lemma 6 , we obtain the following upper bound of $M_{N}$.

Proposition 5. Let $G$ be a graph with $n$ vertices. Also consider

$$
\begin{gathered}
\Delta_{N}=\max \left\{\delta_{G}(v): v \in V(G)\right\}, \\
\delta_{N}=\min \left\{\delta_{G}(v): v \in V(G)\right\} .
\end{gathered}
$$

Then we have

$$
M_{N}(G) \leq \frac{M_{1}^{2}(G)\left(\delta_{N}+\Delta_{N}\right)^{2}}{4 n \delta_{N} \Delta_{N}}
$$

where equality holds iff $\delta_{N}=\Delta_{N}$ or $q=\frac{\frac{\Delta_{N}}{\delta_{N}}}{\frac{\Delta_{N}}{\delta_{N}}+1} n$ is an integer and $q$ of the numbers $x_{i}$ coincide with $\delta_{N}$ and the remaining $(n-q)$ of the $x_{i}^{\prime}$ s coincide with $\Delta_{N}\left(\neq \delta_{N}\right)$. 
Lemma 7 (5). Let $\vec{x}=\left(x_{1}, x_{2}, \ldots, x_{n}\right)$ and $\vec{y}=\left(y_{1}, y_{2}, \ldots, y_{n}\right)$ be sequences of real numbers. Also let $\vec{z}=\left(z_{1}, z_{2}, \ldots, z_{n}\right)$ and $\vec{w}=\left(w_{1}, w_{2}, \ldots, w_{n}\right)$ be non-negative sequences. Then

$$
\sum_{i=1}^{n} w_{i} \sum_{i=1}^{n} z_{i} x_{i}^{2}+\sum_{i=1}^{n} z_{i} \sum_{i=1}^{n} w_{i} y_{i}^{2} \geq 2 \sum_{i=1}^{n} z_{i} x_{i} \sum_{i=1}^{n} w_{i} y_{i}
$$

in particular, if $z_{i}$ and $w_{i}$ are positive, then the equality holds iff $\vec{x}=\vec{y}=\vec{k}$, where $\vec{k}=(k, k, \ldots, k)$, a constant sequence.

Proposition 6. For a graph $G$ with $n$ vertices and $m$ edges, we have

$$
M_{N}(G)>\frac{4 m-n}{n} M_{1}(G)
$$

Proof 3. Considering $x_{i}=\delta_{G}\left(v_{i}\right), y_{i}=\operatorname{deg}_{G}\left(v_{i}\right), z_{i}=1, w_{i}=1$ in lemma 7, we have

$$
\sum_{i=1}^{n} 1 \sum_{i=1}^{n} \delta_{G}\left(v_{i}\right)^{2}+\sum_{i=1}^{n} 1 \sum_{i=1}^{n} \operatorname{deg}_{G}\left(v_{i}\right)^{2} \geq 2 \sum_{i=1}^{n} \operatorname{deg}_{G}\left(v_{i}\right) \sum_{i=1}^{n} \delta_{G}\left(v_{i}\right) .
$$

Now applying definition of $M_{N}$ index, Handshaking lemma and inequality (2.17), we have the following result

$$
M_{N}(G) \geq \frac{4 m-n}{n} M_{1}(G)
$$

From lemma 7 , it is clear that equality in (2.18) is impossible. Hence the proof.

\section{Neighborhood Zagreb index of graph operations}

In this section, we discuss about different graph operations namely join, corona product, strong product, splice, link, disjunction and symmetric difference of graphs and explore the neighborhood Zagreb index for those operations. We start with the following obvious lemma.

Lemma 8. For a graph $G$, we have
(i) $\sum_{v \in V(G)} \delta_{G}(v)=M_{1}(G)$,
(ii) $\sum_{v \in V(G)} \operatorname{deg}_{G}(v) \delta_{G}(v)=2 M_{2}(G)$. 


\subsection{Join}

The join [15] of $G_{1}$ and $G_{2}$ having $V_{1}$ and $V_{2}$ as disjoint vertex sets, is the graph $G_{1}+G_{2}$ which contains $V_{1} \cup V_{2}$ as vertex set and $E_{1} \cup E_{2} \cup$ $\left\{u v: u \in V_{1}, v \in V_{2}\right\}$ as edge set. Clearly we have the following lemma.

Lemma 9. For join of two graphs $G_{1}$ and $G_{2}$, we have

$$
\delta_{G_{1}+G_{2}}(u)=\left\{\begin{array}{l}
n_{2} \operatorname{deg}_{G_{1}}(u)+\delta_{G_{1}}(u)+n_{1} n_{2}+2 m_{2}, \text { if } u \in V_{1} \\
n_{1} \operatorname{deg}_{G_{2}}(u)+\delta_{G_{2}}(u)+n_{1} n_{2}+2 m_{1}, \text { if } u \in V_{2} .
\end{array}\right.
$$

Now we obtain the neighborhood Zagreb index of join of two graphs.

Proposition 7. The neighborhood Zagreb index of $G_{1}+G_{2}$ is given by

$$
\begin{aligned}
M_{N}\left(G_{1}+G_{2}\right)= & n_{1}^{2} n_{2}^{2}\left[n_{1}+n_{2}\right]+8 n_{1} n_{2}\left[n_{1} m_{2}+n_{2} m_{1}\right]+8 m_{1} m_{2}\left[n_{1}+n_{2}\right]+ \\
& 4\left[n_{1} m_{2}^{2}+n_{2} m_{1}^{2}\right]+M_{N}\left(G_{1}\right)+M_{N}\left(G_{2}\right)+\left[n_{1}^{2} M_{1}\left(G_{2}\right)+\right. \\
& \left.n_{2}^{2} M_{1}\left(G_{1}\right)\right]+2 n_{1} n_{2}\left[M_{1}\left(G_{1}\right)+M_{1}\left(G_{2}\right)\right]+4\left[n_{1} M_{2}\left(G_{2}\right)+\right. \\
& \left.n_{2} M_{2}\left(G_{1}\right)\right]+4\left[m_{1} M_{1}\left(G_{2}\right)+m_{2} M_{1}\left(G_{1}\right)\right] .
\end{aligned}
$$

Proof 4. From the definition of neighborhood Zagreb index and using lemma 9, we have

$$
\begin{aligned}
M_{N}\left(G_{1}+G_{2}\right)= & \sum_{u \in V_{1} \cup V_{2}} \delta_{G_{1}+G_{2}}^{2}(u) \\
= & \sum_{u \in V_{1}} \delta_{G_{1}+G_{2}}^{2}(u)+\sum_{u \in V_{2}} \delta_{G_{1}+G_{2}}^{2}(u) \\
= & \sum_{u \in V_{1}}\left[n_{2} \operatorname{deg}_{G_{1}}(u)+\delta_{G_{1}}(u)+n_{1} n_{2}+2 m_{2}\right]^{2} \\
& +\sum_{u \in V_{2}}\left[n_{1} \operatorname{deg}_{G_{2}}(u)+\delta_{G_{2}}(u)+n_{1} n_{2}+2 m_{1}\right]^{2} \\
= & Z_{1}+Z_{2} .
\end{aligned}
$$

Where $Z_{1}$ and $Z_{2}$ are the results of the terms above orderly. Then $Z_{1}$ and $Z_{2}$ will be computed individually. Now,

$$
\begin{aligned}
Z_{1}= & \sum_{u \in V_{1}}\left[n_{2} \operatorname{deg}_{G_{1}}(u)+\delta_{G_{1}}(u)+n_{1} n_{2}+2 m_{2}\right]^{2} \\
= & \sum_{u \in V_{1}}\left[n_{1}^{2} n_{2}^{2}+4 m_{2}^{2}+\delta_{G_{1}}(u)^{2}+\operatorname{deg}_{G_{1}}(u)^{2} n_{2}^{2}+4 n_{1} n_{2} m_{2}+2 n_{1} n_{2} \delta_{G_{1}}(u)+\right. \\
& \left.2 n_{1} n_{2}^{2} \operatorname{deg}_{G_{1}}(u)+4 \delta_{G_{1}}(u) m_{2}+4 \operatorname{deg}_{G_{1}}(u) n_{2} m_{2}+2 \operatorname{deg}_{G_{1}}(u) \delta_{G_{1}}(u) n_{2}\right] .
\end{aligned}
$$


Applying lemma 8, we get

$$
\begin{aligned}
Z_{1}= & n_{1}^{3} n_{2}^{2}+4 n_{1} m_{2}^{2}+M_{N}\left(G_{1}\right)+n_{2}^{2} M_{1}\left(G_{1}\right)+4 n_{1}^{2} n_{2} m_{2}+2 n_{1} n_{2} M_{1}\left(G_{1}\right) \\
& +4 n_{1} n_{2}^{2} m_{1}+4 m_{2} M_{1}\left(G_{1}\right)+8 m_{1} n_{2} m_{2}+4 n_{2} M_{2}\left(G_{1}\right) .
\end{aligned}
$$

Similarly,

$$
\begin{aligned}
Z_{2}= & \sum_{u \in V_{2}}\left[n_{1} \operatorname{deg}_{G_{2}}(u)+\delta_{G_{2}}(u)+n_{1} n_{2}+2 m_{1}\right]^{2} \\
= & n_{1}^{2} n_{2}^{3}+4 n_{2} m_{1}^{2}+M_{N}\left(G_{2}\right)+n_{1}^{2} M_{1}\left(G_{2}\right)+4 n_{1} n_{2}^{2} m_{1}+2 n_{1} n_{2} M_{1}\left(G_{2}\right) \\
& +4 n_{1}^{2} n_{2} m_{2}+4 m_{1} M_{1}\left(G_{2}\right)+8 m_{1} n_{1} m_{2}+4 n_{1} M_{2}\left(G_{2}\right) .
\end{aligned}
$$

Addition of $Z_{1}$ and $Z_{2}$ yield the required result.

Example 1. We can express the complete bipartite graph $\left(K_{n, t}\right)$ as the join of $\bar{K}_{n}$ and $\bar{K}_{t}$. So the above proposition gives $M_{n}\left(K_{n, t}\right)=n^{2} t^{2}(n+t)$.

By suspension of any graph $G$, we mean the join of $G$ and a single vertex $\left(K_{1}\right)$. So we have the corollary stated below followed directly from the above proposition.

Corollary 1. The neighborhood Zagreb index for suspension of a graph $G$ is given by

$$
\begin{aligned}
M_{N}\left(G+K_{1}\right)= & |V(G)|^{2}(|V(G)|+1)+8|V(G)||E(G)|+4|E(G)|^{2}+M_{N}(G) \\
& +M_{1}(G)[1+2|V(G)|]+4 M_{2}(G) .
\end{aligned}
$$

Example 2. The suspension of $\bar{K}_{n}$ produces the star graph $S_{n}$ having ( $n+$ 1) vertices (Figure 1). So its neighborhood Zagreb index can be computed using the above corollary as follows:

$$
M_{N}\left(S_{n}\right)=n^{2}(n+1) .
$$

Example 3. The suspension of $C_{n}$ yields the wheel graph $W_{n}$ containing $n+1$ vertices (Figure 1). So by the above corollary the neighborhood Zagreb index of $W_{n}$ is given by

$$
M_{N}\left(W_{n}\right)=n^{3}+21 n^{2}+36 n .
$$

Example 4. Considering the suspension of $P_{n}$, we get the Fan graph $F_{n}$ having $(n+1)$ vertices (Figure 1$)$. So using the corollary above, we can derive the following result.

$$
M_{N}\left(F_{n}\right)=n^{3}+21 n^{2}+8 n-72, n \geq 4 .
$$




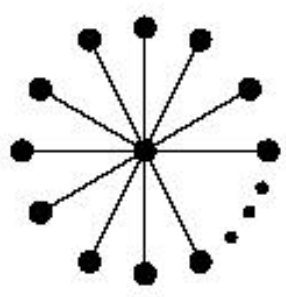

$S_{n}$

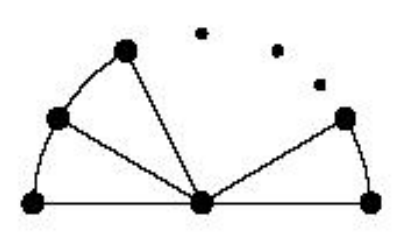

$F_{n}$

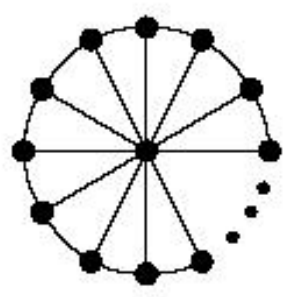

$W_{n}$

Figure 3.1: The example of star, fan and wheel graphs on $n+1$ vertices.

\subsection{Corona product}

For corona product [17] of $G_{1}$ and $G_{2}$, firstly one copy of $G_{1}$ and $n_{1}$ copies of $G_{2}$ are taken and then each node of ith copy of $G_{2}$ is joined to ith node of $G_{1}$, where $1 \leq i \leq n_{1}$. For such operation we denote ith copy of $G_{2}$ as $G_{2, i}$ and ith vertex of $G_{1}$ as $u_{i}$. We can state the following lemma.

Lemma 10. For corona product of two graphs $G_{1}, G_{2}$, we have

$$
\delta_{G_{1} \odot G_{2}}(u)=\left\{\begin{array}{l}
\delta_{G_{1}}(u)+3 n_{2}+2 m_{2}, \text { if } u \in V\left(G_{1}\right) \\
\delta_{G_{2, i}}(u)+\operatorname{deg}_{G_{2, i}}(u)+\operatorname{deg}_{G_{1}}\left(u_{i}\right)+n_{2}, \text { if } u \in V\left(G_{2, i}\right) .
\end{array}\right.
$$

We obtain $M_{N}$ index of corona product of two graphs in the following proposition.

Proposition 8. The neighborhood Zagreb index for corona product is given by

$$
\begin{aligned}
M_{N}\left(G_{1} \odot G_{2}\right)= & M_{N}\left(G_{1}\right)+n_{1} M_{N}\left(G_{2}\right)+\left(7 n_{2}+4 m_{2}\right) M_{1}\left(G_{1}\right)+\left(n_{1}+2 n_{1} n_{2}+\right. \\
& \left.4 m_{1}\right) M_{1}\left(G_{2}\right)+4 n_{1} M_{2}\left(G_{2}\right)+n_{1} n_{2}^{3}+9 n_{1} n_{2}^{2}+4 n_{1} m_{2}^{2}+4 n_{2}^{2} m_{1} \\
& +16 n_{1} n_{2} m_{2}+8 m_{1} m_{2} .
\end{aligned}
$$


Proof 5. Applying the definition of neighborhood Zagreb index and using lemma 10, we have

$$
\begin{aligned}
M_{N}\left(G_{1} \odot G_{2}\right)= & \sum_{v \in V\left(G_{1} \odot G_{2}\right)} \delta_{G_{1} \odot G_{2}}(v)^{2} \\
= & \sum_{v \in V\left(G_{1}\right)} \delta_{G_{1} \odot G_{2}}(v)^{2}+\sum_{i=1}^{n_{1}} \sum_{v \in V\left(G_{2, i}\right)} \delta_{G_{1} \odot G_{2}}(v)^{2} \\
= & \sum_{v \in V\left(G_{1}\right)}\left(\delta_{G_{1}}(v)+2 m_{2}+3 n_{2}\right)^{2} \\
& \left.+\sum_{i=1}^{n_{1}} \sum_{v \in V\left(G_{2, i}\right)}\left(\delta_{G_{2, i}}(v)+\operatorname{deg}_{G_{2, i}}(v)\right)+\operatorname{deg}_{G_{1}}\left(v_{i}\right)+n_{2}\right)^{2} \\
= & C_{1}+C_{2} .
\end{aligned}
$$

Where $C_{1}$ and $C_{2}$ are the above sums orderly. So applying lemma 8, we have

$$
\begin{aligned}
C_{1}= & \sum_{v \in V\left(G_{1}\right)}\left[\delta_{G_{1}}(v)^{2}+6 n_{2} \delta_{G_{1}}(v)+4 m_{2} \delta_{G_{1}}(v)+9 n_{2}^{2}+12 n_{2} m_{2}+4 m_{2}^{2}\right] \\
= & M_{N}\left(G_{1}\right)+\left(6 n_{2}+4 m_{2}\right) M_{1}\left(G_{1}\right)+9 n_{1} n_{2}^{2}+4 n_{1} m_{2}^{2}+12 n_{1} n_{2} m_{2} . \\
C_{2}= & \sum_{i=1}^{n_{1}} \sum_{v \in V\left(G_{2, i}\right)}\left[\delta_{G_{2, i}}(v)^{2}+\operatorname{deg}_{G_{2, i}}(v)^{2}+\operatorname{deg}_{G_{1}}\left(v_{i}\right)^{2}+n_{2}^{2}+2 \delta_{G_{2, i}}(v) \operatorname{deg}_{G_{2, i}}(v)\right. \\
& 2 \delta_{G_{2, i}}(v) \operatorname{deg}_{G_{1}}\left(v_{i}\right)+2 n_{2} \delta_{G_{2, i}}(v)+2 \operatorname{deg}_{G_{2, i}}(v) \operatorname{deg}_{G_{1}}\left(v_{i}\right)+2 n_{2} \operatorname{deg}_{G_{2, i}}(v)+ \\
& \left.2 n_{2} \operatorname{deg}_{G_{1}}\left(v_{i}\right)\right] \\
= & n_{1} M_{1}\left(G_{2}\right)+n_{1} M_{N}\left(G_{2}\right)+n_{2} M_{1}\left(G_{1}\right)+n_{1} n_{2}^{3}+4 n_{1} M_{2}\left(G_{2}\right)+8 m_{1} m_{2}+ \\
& 4 n_{1} n_{2} m_{2}+4 m_{1} M_{1} G_{2}+2 n_{1} n_{2} M_{1}\left(G_{2}\right)+4 n_{2}^{2} m_{1} .
\end{aligned}
$$

Adding $C_{1}$ and $C_{2}$, we obtain the required result.

Corollary 2. The bottleneck graph of a graph $G$ is nothing but the corona product of $K_{2}$ and $G$. By the proposition 8, $M_{N}$ index of this graph is derived bellow.

$$
\begin{aligned}
M_{N}\left(K_{2} \odot G\right)= & 2 M_{N}(G)+(6+4|V(G)|) M_{1}(G)+8 M_{2}(G)+2|V(G)|^{3}+22|V(G)|^{2} \\
& +8|E(G)|^{2}+32|V(G)||E(G)|+14|V(G)|+16|E(G)|+2 .
\end{aligned}
$$


Corollary 3. The $t$-fold bristled graph $\operatorname{Brs}_{t}(G)$ of a graph $G$ is obtained by the corona product of $G$ and $\bar{K}_{t}$. Such type of graph is also called as t-throny graph. Using the proposition 8 , the $M_{N}$ index of t-throny graph is evaluated here.

$M_{N}\left(G \odot \bar{K}_{t}\right)=M_{N}(G)+7 t M_{1}(G)+t^{3}|V(G)|+9 t^{2}|V(G)|+4 t^{2}|E(G)|$.

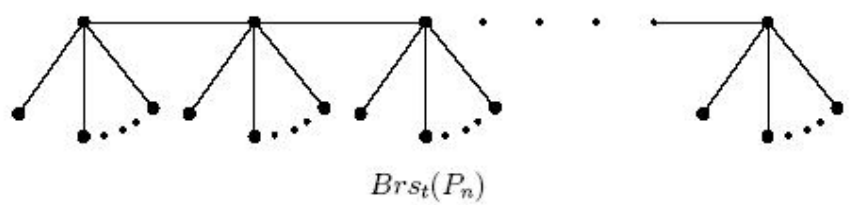

Figure 3.2: The t-throny graphs of $P_{n}$.

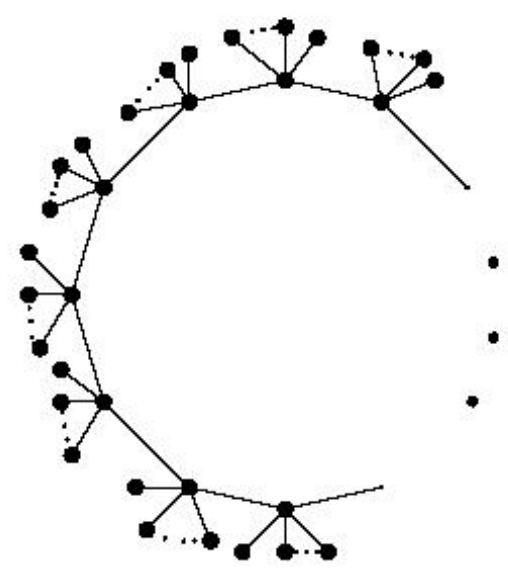

$\mathrm{Brs}_{t}\left(C_{n}\right)$

Figure 3.3: The t-throny graph of $C_{n}$. 
Example 5. The neighborhood Zagreb index of t-throny graph of path (figure 2) and cycle (figure 3 ) graphs on $n$ vertices are computed bellow.

(i) $M_{N}\left(P_{n} \odot \bar{K}_{t}\right)=n t^{3}+13 n t^{2}-4 t^{2}+28 n t+16 n-42 t-38, n \geq 4$.

(ii) $M_{N}\left(C_{n} \odot \bar{K}_{t}\right)=n t^{3}+13 n t^{2}+28 n t+16 n, n \geq 3$.

The $M_{N}$ index of complete bipartite graph, $t$-throny graph of path and cycle graphs are depicted in Figure 3.4.
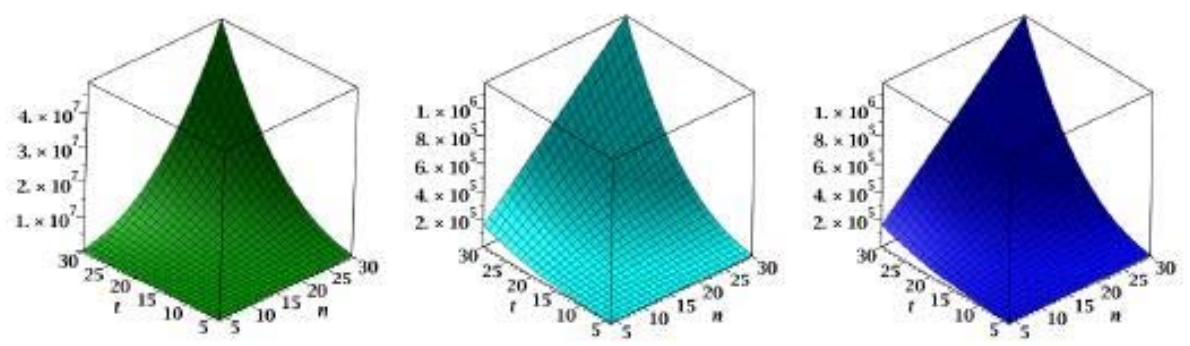

Figure 3.4: Plotting of $M_{N}$ indices for $K_{n, t}, B r s_{t}\left(P_{n}\right)$ and $B r s_{t}\left(C_{n}\right)$ from left to right respectively.

\subsection{Strong product}

The strong product [8] $G_{1} G_{2}$ of $G_{1}, G_{2}$ is a graph with node set $V_{1} \times V_{2}$ and $\left(u_{1}, v_{1}\right)$ is adjacent with $\left(u_{2}, v_{2}\right)$ iff $\left[u_{1}=u_{2}\right.$ and $\left.v_{1} v_{2} \in E_{2}\right]$ or $\left[v_{1}=v_{2}\right.$ and $\left.u_{1} u_{2} \in E_{1}\right]$ or $\left[u_{1} u_{2} \in E_{1}\right.$ and $\left.v_{1} v_{2} \in E_{2}\right]$. Clearly we have the following lemma. 
Lemma 11. For strong product of $G_{1}, G_{1}$, we have

$$
\begin{aligned}
\delta_{G_{1} G_{2}}(u, v)= & \delta_{G_{1}}(u)+\delta_{G_{2}}(v)+\delta_{G_{1}}(u) \delta_{G_{2}}(v)+2 \operatorname{deg}_{G_{1}}(u) \delta_{G_{2}}(v) \\
& +2 \operatorname{deg}_{G_{2}}(v) \delta_{G_{1}}(u)+2 \operatorname{deg}_{G_{1}}(u) \operatorname{deg}_{G_{2}}(v),
\end{aligned}
$$

where $u \in V\left(G_{1}\right), v \in V\left(G_{2}\right)$.

We obtain the neighborhood Zagreb index of strong product of two graphs in the following proposition.

Proposition 9. The neighborhood Zagreb index of strong product of two graphs is given by

$$
\begin{aligned}
M_{N}\left(G_{1} G_{2}\right)= & \left(n_{2}+8 m_{2}\right) M_{N}\left(G_{1}\right)+\left(n_{1}+8 m_{1}\right) M_{N}\left(G_{2}\right)+M_{N}\left(G_{1}\right) M_{N}\left(G_{2}\right)+ \\
& 6\left[M_{1}\left(G_{1}\right) M_{N}\left(G_{2}\right)+M_{N}\left(G_{1}\right) M_{1}\left(G_{2}\right)\right]+8\left[M_{2}\left(G_{1}\right) M_{N}\left(G_{2}\right)+\right. \\
& \left.M_{N}\left(G_{1}\right) M_{2}\left(G_{2}\right)\right]+16\left[m_{2} M_{2}\left(G_{1}\right)+m_{1} M_{2}\left(G_{2}\right)\right]+6 M_{1}\left(G_{1}\right) \\
& M_{1}\left(G_{2}\right)+48 M_{2}\left(G_{1}\right) M_{2}\left(G_{2}\right)+24 M_{1}\left(G_{1}\right) M_{2}\left(G_{2}\right)+24 M_{2}\left(G_{1}\right) M_{1}\left(G_{2}\right) .
\end{aligned}
$$

Proof 6. The proof is directly followed from the definition of neighborhood Zagreb index and lemma 8 and lemma 11.

\subsection{Splice}

The splice [10] of $G_{1}$ and $G_{2}$ by nodes $v_{1} \in V_{1}$ and $v_{2} \in V_{2}$, denoted by $\left(G_{1} \cdot G_{2}\right)\left(v_{1}, v_{2}\right)$, is constructed by identifying the vertices $v_{1}$ and $v_{2}$ in $G_{1} \cup G_{2}$. Thus we have the following lemma.

Lemma 12. For splice of two graphs, we have

$\delta_{\left(G_{1}, G_{2}\right)\left(v_{1}, v_{2}\right)}(u)=\left\{\begin{array}{l}\delta_{G_{1}}\left(v_{1}\right)+\delta_{G_{2}}\left(v_{2}\right), \text { if } u=v_{i}, v_{i} \in V\left(G_{i}\right), i=1,2 \\ \delta_{G_{i}}(u)+\operatorname{deg}_{G_{j}}\left(v_{j}\right), \text { if } u \in N_{G_{i}}\left(v_{i}\right), i \neq j, i, j=1,2 \\ \delta_{G_{i}}(u), \text { if } u \in V\left(G_{i}\right), u \notin N_{G_{i}}\left(v_{i}\right), u \neq v_{i}, i=1,2 .\end{array}\right.$

We calculate the newly introduced index in the following proposition.

Proposition 10. The neighborhood Zagreb index of splice of two graphs is given by

$$
\begin{aligned}
M_{N}\left(\left(G_{1} \cdot G_{2}\right)\left(v_{1}, v_{2}\right)\right)= & M_{N}\left(G_{1}\right)+M_{N}\left(G_{2}\right)+\left|N_{G_{1}}\left(v_{1}\right)\right| \operatorname{deg}_{G_{2}}\left(v_{2}\right)^{2} \\
& +\left|N_{G_{2}}\left(v_{2}\right)\right| \operatorname{deg}_{G_{1}}\left(v_{1}\right)^{2}+2\left[\operatorname{deg}_{G_{2}}\left(v_{2}\right) \sum_{u \in N_{G_{1}}\left(v_{1}\right)} \delta_{G_{1}}(u)\right. \\
& \left.+\operatorname{deg}_{G_{1}}\left(v_{1}\right) \sum_{u \in N_{G_{2}}\left(v_{2}\right)} \delta_{G_{2}}(u)\right]+2 \delta_{G_{1}}\left(v_{1}\right) \delta_{G_{2}}\left(v_{2}\right) .
\end{aligned}
$$


Proof 7. Applying the definition of neighborhood Zagreb index and using lemma 12, we have

$$
\begin{aligned}
M_{N}\left(\left(G_{1} \cdot G_{2}\right)\left(v_{1}, v_{2}\right)\right)= & \sum_{u \in V\left(\left(G_{1} \cdot G_{2}\right)\left(v_{1}, v_{2}\right)\right)} \delta_{\left(G_{1} \cdot G_{2}\right)\left(v_{1}, v_{2}\right)}(u)^{2} \\
= & \left(\delta_{G_{1}}\left(v_{1}\right)+\delta_{G_{2}}\left(v_{2}\right)\right)^{2}+\sum_{u \in N_{G_{i}}\left(v_{i}\right), i \neq j, i, j=1,2}\left[\delta_{G_{i}}(u)+\operatorname{deg}_{G_{j}}\left(v_{j}\right)\right]^{2} \\
& +\sum_{u \in V\left(G_{i}\right), u \notin N_{G_{i}}\left(v_{i}\right), u \neq v_{i}, i=1,2} \delta_{G_{i}}(u)^{2} \\
= & M_{N}\left(G_{1}\right)+M_{N}\left(G_{2}\right)+\left|N_{G_{1}}\left(v_{1}\right)\right| \operatorname{deg}_{G_{2}}\left(v_{2}\right)^{2}+\left|N_{G_{2}}\left(v_{2}\right)\right| \operatorname{deg}_{G_{1}}\left(v_{1}\right)^{2} \\
& +2\left[\operatorname{deg}_{G_{2}}\left(v_{2}\right) \sum_{u \in N_{G_{1}}\left(v_{1}\right)} \delta_{G_{1}}(u)+\operatorname{deg}_{G_{1}}\left(v_{1}\right) \sum_{u \in N_{G_{2}}\left(v_{2}\right)} \delta_{G_{2}}(u)\right]+ \\
& 2 \delta_{G_{1}}\left(v_{1}\right) \delta_{G_{2}}\left(v_{2}\right) .
\end{aligned}
$$

Hence the proof.

\subsection{Link}

The link [10] of $G_{1}, G_{2}$ by nodes $v_{1} \in V_{1}$ and $v_{2} \in V_{2}$, written as $\left(G_{1} \sim\right.$ $\left.G_{2}\right)\left(v_{1}, v_{2}\right)$, is defined by joining the nodes $v_{1}$ and $v_{2}$ with an edge in $G_{1} \cup G_{2}$. The following lemma is clear from above definition.

Lemma 13. For link of two graphs $G_{1}, G_{2}$ by nodes $v_{1} \in V_{1}$ and $v_{2} \in V_{2}$, we have

$\delta_{\left(G_{1} \sim G_{2}\right)\left(v_{1}, v_{2}\right)}(u)=\left\{\begin{array}{l}\delta_{G_{i}}\left(v_{i}\right)+\operatorname{deg}_{G_{j}}\left(v_{j}\right)+1, \text { if } u=v_{i}, v_{i} \in V\left(G_{i}\right), i \neq j, i, j=1,2 \\ \delta_{G_{i}}(u)+1, \text { if } u \in N_{G_{i}}\left(v_{i}\right), i=1,2 \\ \delta_{G_{i}}(u), \text { if } u \in V\left(G_{i}\right), u \notin N_{G_{i}}\left(v_{i}\right), u \neq v_{i}, i=1,2 .\end{array}\right.$

The $M_{N}$ index for link of graphs is obtained in the following proposition.

Proposition 11. The neighborhood Zagreb index for link of two graphs is given by

$$
\begin{aligned}
M_{N}\left(\left(G_{1} \sim G_{2}\right)\left(v_{1}, v_{2}\right)\right)= & M_{N}\left(G_{1}\right)+M_{N}\left(G_{2}\right)+\left[\operatorname{deg}_{G_{1}}\left(v_{1}\right)^{2}+\operatorname{deg}_{G_{2}}\left(v_{2}\right)^{2}\right] \\
& +3\left[\operatorname{deg}_{G_{1}}\left(v_{1}\right)+\operatorname{deg}_{G_{2}}\left(v_{2}\right)\right]+2\left[\delta_{G_{1}}\left(v_{1}\right)+\delta_{G_{2}}\left(v_{2}\right)\right] \\
& +2\left[\delta_{G_{1}}\left(v_{1}\right) \operatorname{deg}_{G_{2}}\left(v_{2}\right)+\delta_{G_{2}}\left(v_{2}\right) \operatorname{deg}_{G_{1}}\left(v_{1}\right)\right] \\
& +2\left[\sum_{u \in N_{G_{1}}\left(v_{1}\right)} \delta_{G_{1}}(u)+\sum_{u \in N_{G_{2}}\left(v_{2}\right)} \delta_{G_{2}}(u)\right]+2 .
\end{aligned}
$$


Proof 8. Applying the definition of neighborhood Zagreb index and using lemma 13, we have

$$
\begin{aligned}
M_{N}\left(\left(G_{1} \sim G_{2}\right)\left(v_{1}, v_{2}\right)\right)= & \sum_{u \in V\left(\left(G_{1} \sim G_{2}\right)\left(v_{1}, v_{2}\right)\right)} \delta_{\left(G_{1} \sim G_{2}\right)\left(v_{1}, v_{2}\right)}(u)^{2} \\
= & \sum_{i \neq j, i, j=1,2}\left[\delta_{G_{i}}\left(v_{i}\right)+\delta_{G_{j}}\left(v_{j}\right)+1\right]^{2}+\sum_{u \in N_{G_{i}}\left(v_{i}\right), i=1,2}\left[\delta_{G_{i}}(u)+1\right]^{2} \\
& +\sum_{u \in V\left(G_{i}\right), u \notin N_{G_{i}}\left(v_{i}\right), u \neq v_{i}, i=1,2} \delta_{G_{i}}(u)^{2} \\
= & M_{N}\left(G_{1}\right)+M_{N}\left(G_{2}\right)+\left[\operatorname{deg}_{G_{1}}\left(v_{1}\right)^{2}+\operatorname{deg}_{G_{2}}\left(v_{2}\right)^{2}\right] \\
& +3\left[\operatorname{deg}_{G_{1}}\left(v_{1}\right)+\operatorname{deg}_{G_{2}}\left(v_{2}\right)\right]+2\left[\delta_{G_{1}}\left(v_{1}\right)+\delta_{G_{2}}\left(v_{2}\right)\right] \\
& +2\left[\delta_{G_{1}}\left(v_{1}\right) \operatorname{deg}_{G_{2}}\left(v_{2}\right)+\delta_{G_{2}}\left(v_{2}\right) \operatorname{deg}_{G_{1}}\left(v_{1}\right)\right] \\
& +2\left[\sum_{u \in N_{G_{1}}\left(v_{1}\right)} \delta_{G_{1}}(u)+\sum_{u \in N_{G_{2}}\left(v_{2}\right)} \delta_{G_{2}}(u)\right]+2 .
\end{aligned}
$$

Hence the proof.

Example 6. Using link of graphs we can obtain the molecular graph of the nanostar dendrimers $D_{n}$ as shown in Figure DC 5. Thus using above proposition we have the following result.

$$
M_{N}\left(D_{n}\right)=624 n-102 .
$$
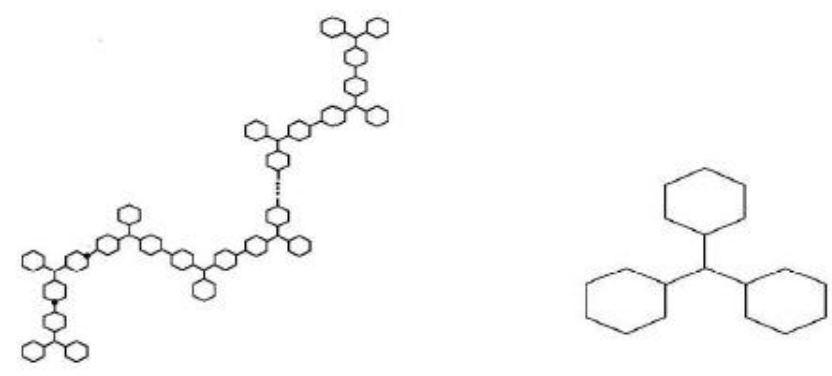

Figure 3.5: The molecular graph of the nanostar dendrimers $D_{n}$ and $D_{1}$. 


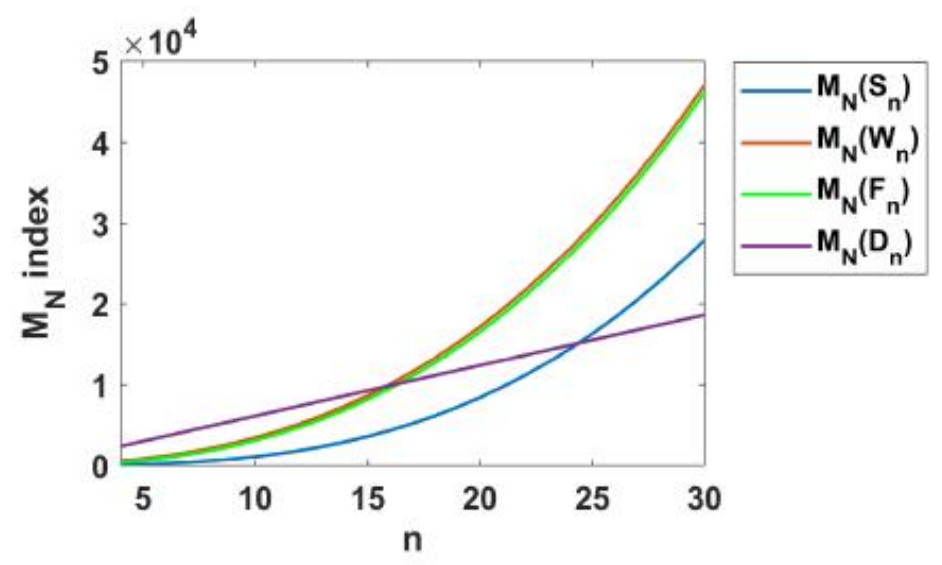

Figure 3.6: Plotting of $M_{N}$ indices for $S_{n}, W_{n}, F_{n}$, and $D_{n}$.

The $M_{N}$ indices of star graph, wheel graph, fan graph and nanostar dendrimer are depicted in Figure 3.6.

\subsection{Disjunction}

The disjunction [15] $G_{1} \vee G_{2}$ of two graphs $G_{1}, G_{2}$ is the graph having node set $V_{1} \times V_{2}$ and $\left(u_{1}, v_{1}\right)$ is adjacent with $\left(u_{2}, v_{2}\right)$ iff $u_{1} u_{2} \in V_{1}$ or $v_{1} v_{2} \in V_{2}$. Clearly we can state the following lemma.

Lemma 14. For disjunction of two graphs

$$
\begin{aligned}
\delta_{G_{1} \vee G_{2}}(u, v)= & \left(n_{2}^{2}-2 m_{2}\right) \delta_{G_{1}}(u)+\left(n_{1}^{2}-2 m_{1}\right) \delta_{G_{2}}(v)+2 n_{2} m_{1} \operatorname{deg}_{G_{2}}(v) \\
& +2 n_{1} m_{2} \operatorname{deg}_{G_{1}}(u)-n_{2} \delta_{G_{1}}(u) \operatorname{deg}_{G_{2}}(v)-n_{1} \operatorname{deg}_{G_{1}}(u) \delta_{G_{2}}(v) \\
& +\delta_{G_{1}}(u) \delta_{G_{2}}(v),
\end{aligned}
$$

where $u \in V\left(G_{1}\right), v \in V\left(G_{2}\right)$.

The $M_{N}$ index for disjunction of two graphs can be computed like previous operations in the following proposition. 
Proposition 12. The neighborhood Zagreb index of disjunction of two graphs is given by

$$
\begin{aligned}
M_{N}\left(G_{1} \vee G_{2}\right)= & {\left[\left(n_{2}^{2}-2 m_{2}\right)\left(n_{2}^{3}-6 n_{2} m_{2}\right)\right] M_{N}\left(G_{1}\right)+\left[\left(n_{1}^{2}-2 m_{1}\right)\left(n_{1}^{3}-6 n_{1} m_{1}\right)\right] } \\
& M_{N}\left(G_{2}\right)+\left[4 n_{1}^{2} n_{2} m_{2}^{2}+8 n_{2} m_{1} m_{2}\left(n_{2}^{2}-2 m_{2}\right)\right] M_{1}\left(G_{1}\right)+\left[4 n_{1} n_{2}^{2} m_{1}^{2}\right. \\
& \left.+8 n_{1} m_{1} m_{2}\left(n_{1}^{2}-2 m_{1}\right)\right] M_{1}\left(G_{2}\right)+\left[8 n_{1} m_{2}\left(n_{2}^{2}-2 m_{2}\right)-16 n_{1} n_{2} m_{2}^{2}\right] \\
& M_{2}\left(G_{1}\right)+\left[8 n_{2} m_{1}\left(n_{1}^{2}-2 m_{1}\right)-16 n_{1} n_{2} m_{1}^{2}\right] M_{2}\left(G_{2}\right)+\left[3 n_{1}^{2}-4 m_{1}\right] \\
& M_{1}\left(G_{1}\right) M_{N}\left(G_{2}\right)+\left[3 n_{2}^{2}-4 m_{2}\right] M_{N}\left(G_{1}\right) M_{1}\left(G_{2}\right)-4\left[n_{1} M_{2}\left(G_{1}\right)\right. \\
& \left.M_{N}\left(G_{2}\right)+n_{2} M_{N}\left(G_{1}\right) M_{2}\left(G_{2}\right)\right]+\left[2\left(n_{2}^{2}-2 m_{2}\right)\left(n_{1}^{2}-2 m_{1}\right)-4 n_{2}^{2} m_{1}\right. \\
& \left.-4 n_{1}^{2} m_{2}\right] M_{1}\left(G_{1}\right) M_{1}\left(G_{2}\right)+\left[16 n_{2} m_{1}-4 n_{1}^{2} n_{2}\right] M_{1}\left(G_{1}\right) M_{2}\left(G_{2}\right)+ \\
& {\left[16 n_{1} m_{2}-4 n_{1} n_{2}^{2}\right] M_{2}\left(G_{1}\right) M_{1}\left(G_{2}\right)+8 n_{1} n_{2} M_{2}\left(G_{1}\right) M_{2}\left(G_{2}\right)+} \\
& M_{N}\left(G_{1}\right) M_{N}\left(G_{2}\right)+32 n_{1} n_{2} m_{1}^{2} m_{2}^{2} .
\end{aligned}
$$

\subsection{Symmetric difference}

The symmetric difference [11] $G_{1} \oplus G_{2}$ of two graphs $G_{1}$ and $G_{2}$ is the graph with node set $V_{1} \times V_{2}$ and edge set $E\left(G_{1} \oplus G_{2}\right)=\left\{\left(u_{1}, v_{1}\right)\left(u_{2}, v_{2}\right): u_{1} u_{2} \in\right.$ $E_{1}$ or $v_{1} v_{2} \in E_{2}$ but not both $\}$. Clearly we have the following lemma.

Lemma 15. For symmetric difference of two graphs

$$
\begin{aligned}
\delta_{G_{1} \oplus G_{2}}(u, v)= & \left(n_{2}^{2}-4 m_{2}\right) \delta_{G_{1}}(u)+\left(n_{1}^{2}-4 m_{1}\right) \delta_{G_{2}}(v)+2 n_{2} m_{1} \operatorname{deg}_{G_{2}}(v) \\
& +2 n_{1} m_{2} \operatorname{deg}_{G_{1}}(u)-2 n_{2} \delta_{G_{1}}(u) \operatorname{deg}_{G_{2}}(v)-2 n_{1} \operatorname{deg}_{G_{1}}(u) \delta_{G_{2}}(v) \\
& +4 \delta_{G_{1}}(u) \delta_{G_{2}}(v),
\end{aligned}
$$

where $u \in V\left(G_{1}\right), v \in V\left(G_{2}\right)$.

In the following proposition, the Neighborhood Zagreb index of symmetric difference of two graphs can be achieved as before.

Proposition 13. The neighborhood Zagreb index of symmetric difference of two graphs is given by

$$
\begin{aligned}
M_{N}\left(G_{1} \oplus G_{2}\right)= & {\left[\left(n_{2}^{2}-4 m_{2}\right)\left(n_{2}^{3}-12 n_{2} m_{2}\right)\right] M_{N}\left(G_{1}\right)+\left[\left(n_{1}^{2}-4 m_{1}\right)\left(n_{1}^{3}-12 n_{1} m_{1}\right)\right] } \\
& M_{N}\left(G_{2}\right)+\left[4 n_{1}^{2} n_{2} m_{2}^{2}+8 n_{2} m_{1} m_{2}\left(n_{2}^{2}-4 m_{2}\right)\right] M_{1}\left(G_{1}\right)+\left[4 n_{1} n_{2}^{2} m_{1}^{2}\right. \\
& \left.+8 n_{1} m_{1} m_{2}\left(n_{1}^{2}-4 m_{1}\right)\right] M_{1}\left(G_{2}\right)+\left[8 n_{1} m_{2}\left(n_{2}^{2}-4 m_{2}\right)-32 n_{1} n_{2} m_{2}^{2}\right] \\
& M_{2}\left(G_{1}\right)+\left[8 n_{2} m_{1}\left(n_{1}^{2}-4 m_{1}\right)-32 n_{1} n_{2} m_{1}^{2}\right] M_{2}\left(G_{2}\right)+\left[12 n_{1}^{2}-\right.
\end{aligned}
$$




$$
\begin{aligned}
& \left.32 m_{1}\right] M_{1}\left(G_{1}\right) M_{N}\left(G_{2}\right)+\left[12 n_{2}^{2}-32 m_{2}\right] M_{N}\left(G_{1}\right) M_{1}\left(G_{2}\right)- \\
& 32\left[n_{1} M_{2}\left(G_{1}\right) M_{N}\left(G_{2}\right)+n_{2} M_{N}\left(G_{1}\right) M_{2}\left(G_{2}\right)\right]+\left[2 ( n _ { 2 } ^ { 2 } - 4 m _ { 2 } ) \left(n_{1}^{2}\right.\right. \\
& \left.\left.-4 m_{1}\right)-8 n_{2}^{2} m_{1}-8 n_{1}^{2} m_{2}\right] M_{1}\left(G_{1}\right) M_{1}\left(G_{2}\right)+\left[64 n_{2} m_{1}-8 n_{1}^{2} n_{2}\right] \\
& M_{1}\left(G_{1}\right) M_{2}\left(G_{2}\right)+\left[64 n_{1} m_{2}-8 n_{1} n_{2}^{2}\right] M_{2}\left(G_{1}\right) M_{1}\left(G_{2}\right)+32 n_{1} n_{2} \\
& M_{2}\left(G_{1}\right) M_{2}\left(G_{2}\right)+16 M_{N}\left(G_{1}\right) M_{N}\left(G_{2}\right)+32 n_{1} n_{2} m_{1}^{2} m_{2}^{2} .
\end{aligned}
$$

\section{Conclusion}

In this article, some bounds of the neighborhood Zagreb index are derived. Also some explicit expressions of the index for different graph operations are obtained and some results are applied to find the index of some composite and chemically interesting graphs. Still there are many other graph operations and special classes of graphs which are not studied here. For further work, the neighborhood Zagreb index of Mycielskis construction, generalized hierarchical product and different subdivision graphs can be obtained.

\section{Acknowledgment}

The first author is very obliged to the Department of Science and Technology (DST), Government of India for the Inspire Fellowship [IF170148].

\section{References}

[1] A. R. Ashrafi, T. Došlić, and A. Hamzeh, "The Zagreb coindices of graph operations", Discrete applied mathematics, vol. 158, no. 15, pp. 15711578, Aug. 2010, doi: 10.1016/j.dam.2010.05.017

[2] M. Azari, "Sharp lower bounds on the Narumi-Katayama index of graph operations", Applied mathematics and computation, vol. 239, pp. 409-421, Jul. 2014, doi: 10.1016/j.amc.2014.04.088

[3] R. Bhatia and C. Davis, "A Better Bound on the Variance", The american mathematical monthly, vol. 107, no. 4, pp. 353-357, Apr. 2000, doi: $10.2307 / 2589180$ 
[4] Z. Che and Z. Chen, "Lower and upper bounds of the forgotten topological index", MATCH communications in mathematical and in computer chemistry, vol. 76, no. 3, pp. 635-648, 2016. [On line]. Available: https://bit.ly/3hvdXD4

[5] K. C. Das, "On geometrical-arithmetic index of graphs", MATCH communications in mathematical and in computer chemistry, vol. 64, no. 3, pp. 619-630, 2010. [On line]. Available: https://bit.ly/2N0M9s]

[6] K. C. Das, A. Yurttas, M. Togan, A. Cevik, and I. Cangul, "The multiplicative Zagreb indices of graph operations", Journal of inequalities and applications, vol. 2013, no. 1, Art ID. 90, Mar. 2013, doi: 10.1186/1029-242x2013-90

[7] N. De, "Some bounds of reformulated Zagreb indices", Applied mathematical sciences, vol. 6, no. 101, pp. 5005-5012, 2012. [On line]. Available: https://bitly/3db09ds

[8] N. De, S. M. A. Nayeem, and A. Pal, "F-Index of some graph operations", Discrete mathematics, algorithms and applications, vol. 8, no. 2, Art ID. 1650025, 2006, doi: 10.1142/s1793830916500257

[9] J. B. Diaz and F. T. Metcalf, "Stronger forms of a class of inequalities of G. Pólya-G. Szegö, and L. V. Kantorovich", Bulletin of the American Mathematical Society, vol. 69, no. 3, pp. 415-419, May 1963, doi: 10.1090/s0002-9904-1963-10953-2

[10] T. Doslić, "Splices, links and their degree-weighted Wiener polynomials", Graph theory notes New York, vol. 48, pp. 47-55, 2005

[11] M. Ghorbani and M. A. Hosseinzadeh, "The third version Of Zagreb index", Discrete mathematics, algorithms and applications, vol. 05, no. 04, Art ID. 1350039, Dec. 2013, doi:10.1142/S1793830913500390

[12] I. Gutman and O. E. Polansky, Mathematical concepts in organic chemistry. Berlin: Springer, 1986, doi: 10.1007/978-3-642-70982-1

[13] I. Gutman and N. Trinajstić, "Graph theory and molecular orbitals. Total $\varphi$-electron energy of alternant hydrocarbons", Chemical physics letters, vol. 17, no. 4, pp. 535-538, Dec. 1972, doi: 10.1016/00092614(72)85099-1

[14] G. H. Hardy, J. E. Littlewood, and P. George, Inequalities, 2nd ed. Cambridge: Cambridge University Press, 1988. 
[15] M. H. Khalifeh, H. Yousefi-Azari, and A. R. Ashrafi, "The first and second Zagreb indices of some graph operations", Discrete applied mathematics, vol. 157, no. 4, pp. 804-811, Feb. 2009, doi:10.1016/j.dam.2008.06.015.

[16] S. Mondal, N. De, and A. Pal, "On neighborhood Zagreb index of product graphs", May 2018, arXiv:1805.05273

[17] K. Pattabiraman and P. Kandan, "Weighted PI index of corona product of graphs", Discrete mathematics, algorithms and applications, vol. 06, no. 04, Art ID.1450055, Oct. 2014, doi: 10.1142/S1793830914500554

[18] G. Pólya and G. Szegö, Problems and theorems in analysis. Berlin: Springer, 1972.

[19] N. Trinajstić, Chemical graph theory. Boca Raton, FL: CRC, 1983.

[20] H. Wiener, "Structural determination of the paraffin boiling points", Journal of the American Chemical Society, vol. 69, no. 1, pp. 17-20, Jan. 1947, doi: 10.1021/ja01193a005 